\title{
Interactions Between Spore Morphogenetic Mutations Affect Cell Types, Sporulation, and Pathogenesis in Magnaporthe grisea
}

\author{
Zhixin Shi, David Christian, and Hei Leung \\ Department of Plant Pathology, Washington State University, Pullman 99164-6430, U.S.A. \\ Accepted 19 November 1997.
}

We have previously defined four single-gene mutations, con1, con2, con4, and con7, that control various stages of spore morphogenesis in the rice blast fungus. To delineate the developmental pathway of spore morphogenesis, we investigated the interactions among these morphogenetic genes by generating strains with double mutations via transformation-mediated gene disruption. Plasmids containing portions of the inactivated $\mathrm{CON} 4$ and $\mathrm{CON} 7$ genes were introduced into strains harboring single mutation to produce double mutants. Interaction between con 1 and con4 resulted in reduced vegetative growth and suppression of sporulation. Interaction between con 1 and con 7 suppressed spore production but not vegetative growth. The con2/con4 and con4/con7 double mutants produced hybrid spore types with characteristic features of both parental mutants. The con $2 / \operatorname{con} 7$ mutant produced $\operatorname{con} 2$ type spores, indicating that con 2 is epistatic to $\operatorname{con} 7$ in spore morphogenesis. The epistatic relationship, however, reversed when traits related to pathogenesis were considered. Double mutants harboring the $\operatorname{con} 7$ mutation could not form appressoria or colonize plant tissue, indicating that $\operatorname{con} 7$ is epistatic to con 2 and con 4 in appressorium formation and pathogenesis. Thus, morphogenetic genes interact at multiple levels leading to different epistatic relationships in the pathways of spore morphogenesis, appressorium formation, and pathogenesis.

Additional keywords: conidia, fungal development, pathogenicity, Pyricularia grisea.

For many plant diseases caused by phytopathogenic fungi, the production of asexual spores is a primary determinant of disease severity. Fungal spores are the infectious propagules responsible for initiating infection as well as for disease dissemination. Interference with critical steps in spore production could be a useful strategy for reducing primary inoculum and the spread of the disease. However, such a strategy has not been widely exploited due to a limited understanding of the genetic regulation of sporulation in phytopathogenic fungi.

Magnaporthe grisea (anamorph, Pyricularia grisea; class: Pyrenomycetes), the causal agent of rice blast disease, has the

Corresponding author: Hei Leung, International Rice Research Institute, P.O. Box 933, Manila, Philippines; Phone: 63-2-845-0563; Fax: 63-2845-1292; E-mail: H.Leung@cgnet.com genetic attributes that make it highly amenable for genetic and molecular analysis (Valent and Chumley 1991). Genetic crosses can be made readily, and efficient transformation systems are available (Parsons et al. 1987; Leung et al. 1990; Shi et al. 1995). The fungus produces abundant asexual spores (conidia) that are responsible for the multiple infection cycles within a cropping season. The sporulation apparatus consists of a conidiophore bearing sympodially 4 to 5 pyriform, three-celled conidia. On media favoring sporulation, the fungus produces spores approximately 2 days after conidial germination (Leung and Shi 1994). On the rice plant, sporulation begins on susceptible lesions approximately 5 days after inoculation.

Using chemical and insertional mutagenesis, we have identified six genes-con1, con2, con4, con5, con6, and con7that control various steps in the sporulation pathway (Shi and Leung 1994; Leung and Shi 1994; Shi and Leung 1995). Each of these mutations results in a unique profile of abnormal characteristics in sporulation and spore morphogenesis. Mutation at the con 5 locus completely blocks conidiophore formation whereas mutation at the con6 locus leads to the production of abundant conidiophores but no conidia. Mutations at the con1, con2, con4, and con 7 loci result in the production of conidia with distinct cell shapes. The con 2 mutant is aconidial in the dark but produces abnormally shaped, one- or twocelled conidia under direct illumination. The conl mutant produces elongated conidia that are twice as long $(50 \mu \mathrm{m})$ and half as wide $(8 \mu \mathrm{m})$ as the wild-type conidia. Conidia of the con4 mutant are ellipsoid with a slight narrowing of the basal cell. The con 7 mutant produces a mixture of normally and abnormally shaped conidia. The characteristic type of conidia is slightly elongated, and many have attenuated tip cells. The con 7 mutant produces equally abundant conidia in total darkness as under direct illumination.

A common feature among four spore-producing mutations, con1, con2, con4, and con7, is a reduction in pathogenicity on rice. Reduced pathogenicity in the con 2 and con 4 mutants is manifested as a lower infection efficiency and longer latent period relative to the wild type (Shi and Leung 1995). Conidia of both con 2 and con 4 mutants produce fewer appressoria than the wild type and cause few lesions on rice plants inoculated with a high concentration of spores. Conidia of the conl and con7 mutants do not produce appressoria and are nonpathogenic on rice (Shi and Leung 1995). Furthermore, the loss of pathogenicity in the conl and con 7 mutants cannot be com- 
pensated by wound inoculation, suggesting that additional developmental defects other than penetration are responsible for the loss of pathogenicity in these mutants. Genetic analyses showed that con 2 is epistatic to con1, con 5 epistatic to con6, and con6 epistatic to con 7 (Shi and Leung 1995); however, the interactions between other morphogenetic genes remain unclear due to infertility in most crosses between mutants.

To further delineate the sporulation pathway, we investigated the interactions between morphogenetic loci with transformation-mediated gene disruption. We report here the construction and analyses of a series of mutants carrying double mutations. We found that interactions between the morphogenetic mutations result in hybrid spore types or a complete suppression of spore formation and pathogenesis.

\section{RESULTS}

Isolation of inactivated genes by plasmid rescue.

Genetic analyses indicated that both con 4 and con 7 genes in GT20 (con4) and GT562 (con7) mutants are tagged by inserted plasmid pAN7-2 (see Table 1 for strain designations; Shi and Leung 1995). To retrieve the mutated CON4 and CON7 genes, mutant genomic DNA was digested with ApaI (no recognition site in pAN7-2) and self-ligated. The ligated DNA was used to transform Escherichia coli by electroporation. Approximately 50 ampicillin resistant colonies were recovered by transforming $10^{8} \mathrm{E}$. coli cells with $0.1 \mu \mathrm{g}$ of ligated genomic DNA. The plasmids retrieved from the con 4 and con 7 mutants were named pGT20A and pGT562A to indicate plasmids containing ApaI-digested fragments from strains GT20 and GT562, respectively. pGT20A contained approximately $1 \mathrm{~kb}$ and $1.2 \mathrm{~kb}$ of flanking sequences on either side of the inserted pAN7-2 (D. Fujimoto, Z. Shi, D. Christian, and H. Leung, unpublished results). pGT562A contained approximately $2.4 \mathrm{~kb}$ of flanking DNA (Z. Shi and H. Leung, unpublished result).

To confirm that the retrieved plasmids represent the inactivated CON4 and CON7 loci, pGT20A and pGT562A were linearized with $A p a \mathrm{I}$ and introduced into wild-type strains by transformation. Mutants with con 4 spore morphology were recovered at a high frequency $(\approx 40 \%)$ from transformation of Guy11 with pGT20A. The phenotypes of mutant transformants were identical to those of the original GT20 mutant with respect to spore morphology, sporulation, and appressorium formation. When pGT562A was introduced into Guy11, $16 \%$ of the transformants ( 8 out of 51) showed typical con 7 mutant spore and colony morphology. The representative con4 and con7 mutant transformants were subjected to Southern analysis with pAN7-2 as a probe. GR17-2 (con4) and GR16-35 (con7) mutant transformants have patterns identical to the original mutants, GT20 and GT562, respectively (Fig. 1A). These results indicate that pGT20A and pGT562A contain at least a portion of the inactivated CON4 and $C O N 7$ genes, respectively.

\section{Production of double mutants by gene disruption.}

Three strains, Guy11-con2, 89-8-1, and GR43-3, carrying the con 2 , con $1-C$, and con 7 mutations, respectively, were used as recipients for transformation. When pGT20A was introduced into Guy11-con2, mutant transformants with nonparental spore morphology were recovered. Of 36 transformants obtained, 14 produced spores that were morphologically distinct from those of either the con 2 or con 4 mutants. The new spore type had morphological features intermediate between the parental mutants (Fig. 2). Most of the hybrid spores were elongated (similar to the ellipsoid conidia of the con 4 mutant) but with a single septum (characteristic of con2).

When pGT562A was introduced into Guy11-con2, a transformant, GR19-8, with new phenotypes was recovered. GR198 produced con 2 type spores but had colony morphology characteristic of the $\operatorname{con} 7$ mutant (erect and fragile conidio-

Table 1. Strains of Magnaporthe grisea and plasmids used in this study

\begin{tabular}{|c|c|c|}
\hline $\begin{array}{l}\text { Strain or } \\
\text { plasmid }\end{array}$ & $\begin{array}{l}\text { Genotypes and relevant } \\
\text { characteristics }\end{array}$ & Reference \\
\hline \multicolumn{3}{|l|}{ M. grisea } \\
\hline Guy11 & MAT-2 & Leung et al. 1988 \\
\hline 2539 & MAT-1 & Leung et al. 1988 \\
\hline $89-8-1$ & $M A T-2$, con $1-C$ & $\begin{array}{l}\text { Gift of A. H. Elling- } \\
\text { boe, Univ. of Wis- } \\
\text { consin-Madison }\end{array}$ \\
\hline Guy11-con2 & $M A T-2, \operatorname{con} 2$ & Leung and Shi 1994 \\
\hline GT20 & MAT-2, con4 & Shi and Leung 1995 \\
\hline GT562 & MAT-2, con 7 & Shi and Leung 1995 \\
\hline GR14-1 & Guy $11-\operatorname{con} 2 /$ CON 4 & This study \\
\hline GR14-2 & Guy11-con2/CON4 & This study \\
\hline GR14-3 & Guy11-con2/con4 & This study \\
\hline GR14-7 & Guy11-con $2 / \operatorname{con} 4$ & This study \\
\hline GR16-3 & Guy11/CON7 & This study \\
\hline GR16-4 & Guy11/CON7 & This study \\
\hline GR16-17 & Guy $11 /$ con 7 & This study \\
\hline GR16-35 & Guy $11 / \operatorname{con} 7$ & This study \\
\hline GR17-1 & Guy11/con4 & This study \\
\hline GR17-3 & Guy11/CON4 & This study \\
\hline GR17-4 & Guy11/CON4 & This study \\
\hline GR19-7 & Guy11-con2/CON7 & This study \\
\hline GR19-8 & Guy11-con2/con7 & This study \\
\hline GR28-9 & con1-C/ CON4 & This study \\
\hline GR28-4 & $\begin{array}{l}\text { con1-C/con4, no spore, re- } \\
\text { duced growth }\end{array}$ & This study \\
\hline GR29-9 & con1-C/CON7 & This study \\
\hline GR29-1 & $\begin{array}{l}\operatorname{con} 1-C / \operatorname{con} 7, \text { no spore, frag- } \\
\text { ile conidiophore }\end{array}$ & This study \\
\hline GR29-8 & $\begin{array}{l}\operatorname{con} 1-C / \operatorname{con} 7, \text { no spore, frag- } \\
\text { ile conidiophore }\end{array}$ & This study \\
\hline GR43-3 & $\begin{array}{l}\text { con7 mutant produced by } \\
\text { gene disruption with } \\
\text { pCON7::bar }\end{array}$ & This study \\
\hline GR44-1 & $\operatorname{con} 4 / \operatorname{con} 7$ & This study \\
\hline GR44-3 & $\operatorname{con} 4 / \operatorname{con} 7$ & This study \\
\hline GR44-10 & CON4/con 7 & This study \\
\hline \multicolumn{3}{|l|}{ Plasmid } \\
\hline pAN7-2 & $\begin{array}{l}\text { 9.2-kb cosmid vector con- } \\
\text { taining Escherichia coli } \\
\text { hygromycin B phos- } \\
\text { photransferase gene }\end{array}$ & Punt et al. 1987 \\
\hline pBARGEM7-2 & $\begin{array}{l}\text { 4.9-kb plasmid containing the } \\
\text { bar gene, which confers } \\
\text { phosphinothricin resi stance }\end{array}$ & $\begin{array}{l}\text { Pall and Brunelli } \\
1993\end{array}$ \\
\hline pGT20A & $\begin{array}{l}\text { Self-ligated plasmid contain- } \\
\text { ing pAN7-2 plus flanking } \\
\text { DNA from the con4 locus } \\
\text { in GT20 }\end{array}$ & This study \\
\hline pGT562A & $\begin{array}{l}\text { Self-ligated plasmid contain- } \\
\text { ing pAN7-2 plus flanking } \\
\text { DNA from the con7 locus } \\
\text { in GT562 }\end{array}$ & This study \\
\hline pCON7::bar & $\begin{array}{l}\text { CON7 cDNA disrupted by in- } \\
\text { sertion of the bar cassette } \\
\text { at } E c o \mathrm{RV} \text { site }\end{array}$ & This study \\
\hline
\end{tabular}


phores), though much reduced in sporulation capacity (similar to the con2 mutant). Unlike Guy11-con2, conidia of GR19-8 germinated poorly and did not produce appressoria (Fig. 2).

Introduction of pGT20A into 89-8-1 (con1-C mutation) generated 24 transformants. One transformant, GR28-4, was aconidial and exhibited reduced radial growth. Two aconidial mutants (GR29-1 and GR29-8) were recovered from 89-8-1 transformed with pGT562A. These two transformants formed abundant conidiophore-like hyphae with little reduction in vegetative growth. The conidiophore-like hyphae appeared to be fragile (a characteristic of the con7 mutant), as many conidiophore fragments were found in suspension of aerial mycelia.

Since both con 4 and con 7 are insertional mutants carrying hygromycin B resistance, a different approach involving an alternate selection marker was used to construct a con $4 /$ con 7 mutant. Cloning of the cDNA corresponding to the CON7 gene allowed us to construct a gene disruption vector, pCON7::bar, in which the CON7 open reading frame was disrupted by the insertion of the bar gene cassette from pBARGEM7-2. pCON7::bar was used to disrupt the CON7 gene in Guy11 to generate con7 mutants with phosphinothricin resistance (Z. Shi and H. Leung, unpublished results). A representative con7 mutant (GR43-3) was used as the recipient for transformation with pGT20A. Eight out of 70 hygromycin B resistant transformants $(11 \%)$ had spore characteristics intermediate between those of the con4 and con 7 mutants (Fig. 2). All transformants with novel spore morphology and/or other phenotypes were considered putative gene

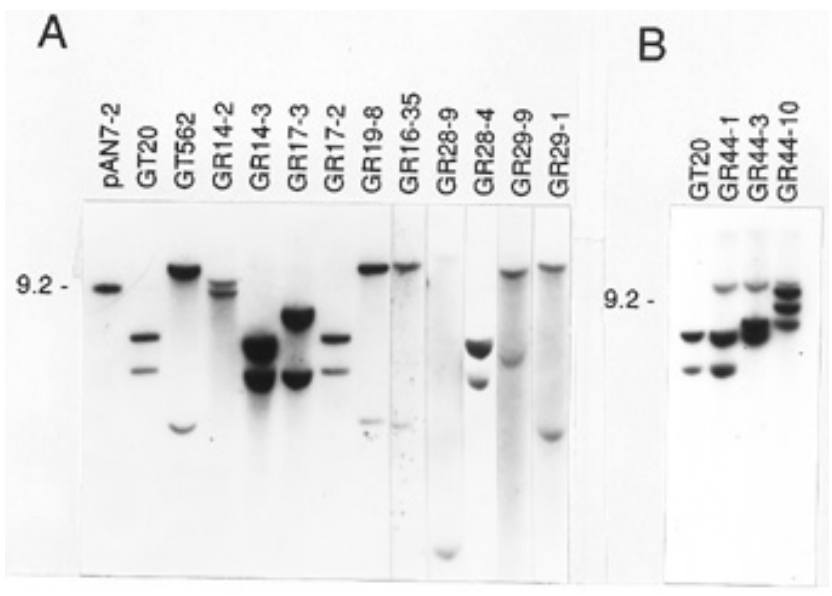

Fig. 1. Southern analysis of double mutants and ectopic integrants of Magnaporthe grisea. Genomic DNA was digested with Bam $\mathrm{HI}$ and electrophoretically separated on $0.7 \%$ agarose gel. Southern blot was probed with pAN7-2. Size in kb of linearized pAN7-2 is indicated on the left side. A, Representative single and double mutants and ectopic integrants generated by introducing plasmids pGT20A or pGT562A into strains Guy11, Guy11-con2, and 89-8-1. Gene replacement mutants (GR) show pattern identical to that of the original mutations: GR14-3 (con2/con4), GR17-2 (con4), GR28-4 (con1/con4) have the same hybridization pattern as GT20; GR19-8 (con2/con7), GR16-35 (con7), GR29-1 (con1/con7) have the same pattern as GT562. The other GR strains are ectopic integrants. B, con $4 / \operatorname{con} 7$ double mutants generated by insertion of pGT20A into con7 mutant strain GR43-3. GT20 and GR441 share two common bands. Uppermost band represents homology between parental vectors pAN7-2 and pBARGEM7-2. Strain GR44-3 is also considered a gene replacement mutation; however, sequence changes may have occurred that disrupted one BamHI restriction site. GR44-10 is an ectopic integrant. disruption mutants and were subjected to genetic and molecular analyses.

\section{Genotypes of double mutants confirmed by genetic and Southern blot analyses.}

To confirm that the transformants with novel phenotypes were caused by double mutations at the respective loci, progeny were obtained from crosses between the double mutants and wild-type strain 2539 . While crosses between two single mutants were sterile (e.g., con $1 \times \operatorname{con} 7$ or $\operatorname{con} 2 \times \operatorname{con} 4)$, crosses between the putative double mutants and 2539 were sufficiently fertile to allow for segregation analysis. No genetic data were obtained from the putative conl/con4 mutant GR28-4 as numerous attempts to cross the mutant to 2539 were not successful.

In crosses involving putative con $2 / \operatorname{con} 4, \operatorname{con} 1 / \operatorname{con} 7$, and con4/con 7 double mutants, all four expected genotypes (two parental and two recombinant types) were recovered in the progeny, confirming that the novel morphologies were the result of double mutations (Table 2). For cross 1559 (con2/con4 $\times 2539$ ), the segregation ratio of the progeny was in close agreement with the expected 1:1:1:1 ratio, indicating that the con 2 and con 4 loci are not genetically linked. In cross 1562 $(\operatorname{con} 1 / \operatorname{con} 7 \times 2539)$, there was an excess of con $1 /$ con 7 and con1/CON7 progeny, which we cannot explain at this time. In crosses 1572 and 1573 (con4/con7 $\times 2539$ ), there was an excess of wild type, which may reflect a reduced viability of the mutant progeny.

Three phenotypic classes were detected among progeny from cross 1581 (GR19-8 × 2539; GR19-8 was derived from transformation of Guy11-con2 with pGT562A): con2, con7, and wild type. The recovery of con 7 progeny confirmed that GR19-8 harbored a con7 mutation. The most frequent category was con 2 progeny, which were further classified according to their sensitivity to hygromycin B. Approximately half of the con 2 progeny were resistant to hygromycin and the other half sensitive. The hygromycin-resistant con 2 progeny produced very few spores, a phenotype similar to the GR19-8 parent. It was inferred that these con 2 progeny have the con $2 / \operatorname{con} 7$ genotype. The segregation ratio of the four genotypes did not deviate significantly from the 1:1:1:1 ratio, which is consistent with the hypothesis that the con 2 mutation is epistatic to $\operatorname{con} 7$ in defining spore morphology and that the two loci are genetically unlinked.

To further confirm the genotypes of these double mutants, genomic DNA of representative transformants and the original mutants were subjected to Southern analysis with pAN7-2 as the hybridization probe. For the con2/con4 (GR14-3), con1/con4 (GR28-4), and con4/con7 (GR44-1) mutants generated by transformation with pGT20A, the hybridization patterns were identical to those of the original con4 mutant (Fig. 1A, compare GR14-3 and GR28-4 with GT20; Fig. 1B, GR44-1 with GT20). Similarly, gene disruptions in the 89-8-1 and Guy11-con2 mutants mediated by transformation with pGT562A gave a hybridization pattern identical to that of the original insertional mutant, GT562 (Fig. 1A, compare GR19-8 and GR29-1 with GT562). The detection of identical flanking DNA fragments indicated that gene replacement events were mediated by a double cross-over between homologous regions of the genome and the introduced plasmids. One exception in the Southern analysis is the pattern of GR44-3 (con4/con7 
double mutant, compare it with GT20 in Figure 1B). It is possible that GR44-3 is the result of gene disruption due to single cross-over. Alternately, the unexpected pattern may be due to sequence changes occurring at one of the BamHI sites in a gene replacement event. Transformants that had phenotypes identical to parental recipient strains were also examined for their integration patterns. In all cases, ectopic integrations were observed (Fig. 1).

Thus, genetic analyses and Southern hybridization patterns together confirmed that all the morphological variants are double mutants caused by gene replacement or disruption events at the targeted loci. The fact that ectopic integrants had spore morphology identical to parental recipient strains indicates that the wild-type alleles of the CON4 and CON7 loci are dominant to the mutant alleles (con4 and $\operatorname{con} 7$ ).

\section{Interactions between morphogenetic loci in appressorium formation and pathogenesis.}

Table 3 summarizes the radial growth and light response of representative double mutants generated by gene disruption or replacement. Ectopic transformants from the corresponding experiments were included as controls. Radial growth was similar in the single and double mutants except for the con1/con4 mutant (GR28-4), which was significantly reduced in vegetative growth. The effect of the con 7 mutation on light response was evident in the con4/con 7 mutants (GR44-1 and
GR44-3), which produced similar amounts of spores with or without light. The light response of the $\operatorname{con} 2 / \operatorname{con} 7$ mutant was similar to that of the con 2 mutant, although the double mutant showed much reduced sporulation.

Appressorium formation of double mutants was evaluated on hydrophobic microscope slide surfaces and on detached leaf sheaths. The detached leaf assay was conducted to test whether signal(s) from plants may play a role in the induction of appressorium formation. As expected, the con $2 /$ con $4 \mathrm{mu}-$ tant formed appressoria since both the parental con 2 and con 4 mutants can form appressoria, albeit at a reduced level (Shi and Leung 1995). No appressoria were formed by the con4/con 7 and con $2 / \operatorname{con} 7$ mutants, indicating that con 7 is epistatic to con 2 and con 4 in appressorium formation. In all cases, the patterns of appressorium formation on slides and leaf sheath were similar. Thus, there was no evidence of inducive factors from the plant that would compensate for the defects in these mutants.

Since cyclic AMP (cAMP) has been shown to induce appressorium formation in M. grisea (Lee and Dean 1993), we evaluated the responses of single and double mutants to exogenous cAMP (Fig. 3). Appressorium formation in the con2 and con 4 mutants was partially responsive to cAMP such that exogenously added cAMP induced appressorium formation on a hydrophilic surface (ethanol-cleaned microscope coverslip). The concentration of cAMP required to induce appressorium

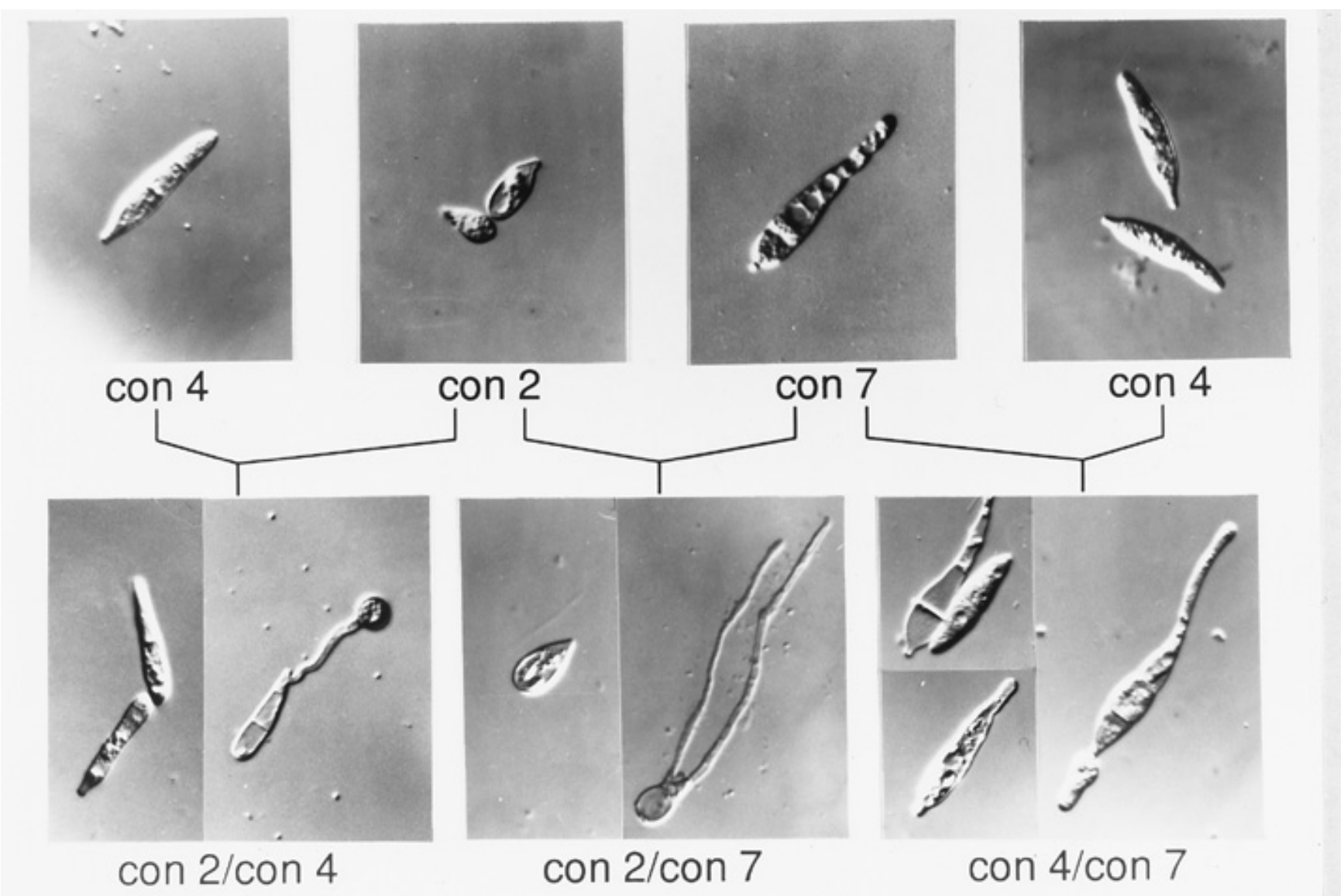

Fig. 2. Conidial morphology of selected single and double mutants of Magnaporthe grisea. Double mutants show hybrid spore morphology. con2/con4 mutant shows a slight narrowing of the basal cell and has only a single septum. con2/con7 mutant produces con2-type conidia that do not form appressoria. con $4 / \operatorname{con} 7$ mutant shows a narrowing of the basal cell and does not form appressoria. con1/con4 and conl/con7 mutants are aconidial (not shown). 
formation in the con 4 mutant was greater than that needed for the wild type. For the con 2 mutant, approximately $50 \%$ of the germ tubes produced appressoria when treated with $50 \mathrm{mM}$ cAMP. No response to cAMP was observed in the con $2 /$ con 4 double mutant even though con 2 and con 4 mutants were partially responsive to cAMP. This would suggest that the defects in response to cAMP were additive in the con $2 /$ con 4 double mutant. The con 1 and con 7 mutants did not form appressoria on a hydrophobic surface and were not responsive to cAMP within the range of concentrations tested. No appressoria were formed by the con $2 / \operatorname{con} 7$ and con4/con 7 double mutants in response to exogenous cAMP, indicating that $\operatorname{con} 7$ is epistatic to con 2 and con 4 in its response to cAMP.

Pathogenicity of the gene replacement mutants and their corresponding ectopic integrants was tested on rice variety M103, which is highly susceptible to the parental strain Guy11 (Table 4). The gene-disrupted con4 and con 7 mutants both exhibited pathogenic properties identical to the original mutants, GT20 and GT562, respectively. For example, GR17-1 (con4) caused only a few lesions at a high inoculum level; GR16-17 and GR16-35 (con7) were nonpathogenic. The con2/con4 mutants (GR14-3 and GR14-7) were weakly pathogenic on M103 as assayed by spray inoculation. The number of lesions produced by the con $2 /$ con 4 mutants was less than that produced by either single-mutation parent (con2 or con4). The con $2 /$ con 7 and con4/con 7 double mutants were nonpathogenic on rice as assayed by spray inoculation (Table 4). The lack of macroscopic symptoms suggests that few, if any, cells were invaded by the double mutants. Pathogenicity of mutants was also assayed by injecting spore suspension into the leaf sheaths of plants. The wound inoculation assay allowed us to test whether the mutants were capable of colonizing the plant tissue if the penetration step was by-passed. The results of wound inoculation were consistent with those of spray inoculation. With the exception of the con $2 /$ con 4 mutant, no double mutants caused lesions on injected leaves (Table 4).

\section{DISCUSSION}

The conventional approach for investigating epistatic relationships among single-gene mutations is to determine the phenotypes of double mutants generated by genetic crosses. Double mutants, however, can be difficult to produce due to reduced fertility of the mutants. Furthermore, the double mutant recovered among segregating progeny has a genetic background different from either parental strain where isogenic strains are not available for genetic analysis, and hence the trait of interest may be masked by other segregating genes. We demonstrate in this report that these problems can be overcome by transformation-mediated gene disruption. This approach will be especially important for some fungi that lack a sexual stage for genetic analysis. We made use of three morphogenetic mutants, con1-C (spontaneous), con2 (chemically induced), and con7 (CON7 gene disrupted in GR43-3), as recipients for transformation. Retrieved plasmids carrying an inactivated gene (con4 or con 7 ) and a selectable marker, hygromycin B phosphotransferase, were used to replace the corresponding wild-type genes. A combination of genetic and molecular analyses confirmed the genotypes of transformants with double mutations. Gene replacement mutants were recovered at a high frequency. All double mutants examined, with the exception of GR44-3 (con4/con7), are the result of gene replacement events, which occur in 4 to $40 \%$ of transformants.

Table 2. Segregation analysis of double mutants of Magnaporthe grisea generated by gene replacement

\begin{tabular}{|c|c|c|c|c|c|c|}
\hline Cross no. & Parents & Parental genotype & Expected progeny genotype & Observed progeny (no.) & $\chi^{2}$ & $P$ value \\
\hline 1559 & GR14-3 × 2539 & $\operatorname{con} 2 / \operatorname{con} 4 \times$ CON $2 /$ CON 4 & $\begin{array}{c}\text { con } 2 / \text { con } 4 \\
\text { CON } 2 / \text { CON } 4 \\
\text { con } 2 / \text { CON } 4 \\
\text { CON } 2 / \text { con } 4\end{array}$ & $\begin{array}{l}20 \\
27 \\
26 \\
26\end{array}$ & 1.24 & $0.75>P>0.5$ \\
\hline 1559B & GR14-7 × 2539 & $\operatorname{con} 2 / \operatorname{con} 4 \times$ CON $2 / C O N 4$ & $\begin{array}{c}\text { con } 2 / \text { con } 4 \\
\text { CON2/CON4 } \\
\text { con } 2 / C O N 4 \\
\text { CON } 2 / \text { con } 4\end{array}$ & $\begin{array}{r}7 \\
11 \\
6 \\
10\end{array}$ & 1.99 & $0.75>P>0.5$ \\
\hline 1581 & GR19-8 × 2539 & $\operatorname{con} 2 / \operatorname{con} 7 \times$ CON $2 / C O N 7$ & $\begin{array}{c}\text { con } 2 / \operatorname{con} 7 \\
\text { CON } 2 / \text { CON7 } \\
\text { con } 2 / \text { CON7 } \\
\text { CON } 2 / \operatorname{con} 7\end{array}$ & $\begin{array}{l}4^{\mathrm{a}} \\
8 \\
7 \\
7^{\mathrm{a}}\end{array}$ & 1.37 & $0.75>P>0.5$ \\
\hline 1562 & GR29-8 × 2539 & con $1 / \operatorname{con} 7 \times$ CON1/CON7 & $\begin{array}{c}\text { con1/con7 } \\
\text { CON1/CON7 } \\
\text { con1/CON7 } \\
\text { CON1/con7 }\end{array}$ & $\begin{array}{r}17 \\
4 \\
14 \\
7\end{array}$ & 14.9 & $0.01>P$ \\
\hline 1572 & GR44-1 × 2539 & con $4 / \operatorname{con} 7 \times$ CON4/CON7 & $\begin{array}{c}\text { con4/con7 } \\
\text { CON4/CON7 } \\
\text { con } 4 / \text { CON7 } \\
\text { CON4/con7 }\end{array}$ & $\begin{array}{l}2 \\
9 \\
1 \\
6\end{array}$ & 9.1 & $0.05>P>0.01$ \\
\hline 1573 & GR44-3 × 2539 & con4/con $7 \times$ CON4/CON7 & $\begin{array}{c}\text { con } 4 / \operatorname{con} 7 \\
\text { CON4/CON7 } \\
\text { con } 4 / \text { CON7 } \\
\text { CON4/con7 }\end{array}$ & $\begin{array}{r}6 \\
18 \\
6 \\
7\end{array}$ & 11.1 & $0.05>P>0.01$ \\
\hline
\end{tabular}

${ }^{\mathrm{a}}$ Hygromycin B resistant. 
Our initial model assumed sporulation to be controlled by a linear pathway such that genes controlling earlier conidial development would be epistatic to those controlling later stages. We have shown by genetic analysis that con 2 is epistatic to conl (Shi and Leung 1995). Considering the underdeveloped conidial morphology of the con 2 mutant, we anticipated that con 2 would be epistatic to both con 4 and con 7 . Results from this study indicate that con 2 is epistatic to con 7 but not to con4, as evidenced by the formation of a hybrid spore type in the con $2 /$ con 4 double mutant. Novel morphogenetic phenotypes were observed in other gene interactions as well. Sporulation was completely suppressed in the conl/con4 and con 1/con 7 mutants and hybrid spores were produced in the con $2 /$ con 4 and con $4 /$ con 7 mutants. The most prominent phenotype of the con 2 mutation is the production of one- or twocelled conidia. Since the morphogenetic abnormality in the con1 and con 7 mutants may only be manifested at the threecelled stage, con 2 becomes epistatic to con 1 and $\operatorname{con} 7$ by blocking the development of a three-celled conidium. Results from this study, together with our previous work (Shi and Leung 1995) indicate the presence of a core pathway (con5> con6>con $2>\operatorname{con} 1$ and con7) determining conidial development in $M$. grisea. However, spore morphology can be modified by other genes functioning independently of this core pathway (e.g., the CON4 gene). The role of CON4 in this sporulation pathway is consistent with the nature of the gene. Preliminary sequence analysis indicates that this gene encodes a membrane-associated glycoprotein (D. Fujimoto, Z. Shi, D. Christian, and H. Leung, unpublished results). It is important to point out that the epistatic relationships described here are entirely based upon on the morphogenetic phenotypes and do not necessarily imply a regulatory relationship as has been demonstrated in A. nidulans (Busby et al. 1996; Miller 1990; Timberlake 1993).

Figure 4A summarizes the different gene interactions occurring during spore development. In the spore morphogenesis pathway, con 2 acts upstream at a branch point before con 1 and con 7 in the determination of spore shape. Interaction between the con 1 and con 7 mutations results in the production of conidiophores only. Interestingly, this conidiophore-only phenotype is similar to that of an upstream mutation, con6, described previously (Shi and Leung 1995). It is possible that developmental blocks in both conl and con 7 are functionally equivalent to a single block in an upstream gene. In this model, con4 functions independently of the core pathway, but exerts a strong influence on spore morphogenesis.

Examination of traits other than spore morphogenesis, however, shows different epistatic relationships among the morphogenetic loci (Table 5). The con 7 mutation is completely epistatic to con 2 and con4 in the formation of appressoria and responsiveness to cAMP. cAMP has been shown to play an important role in the regulation of fungal development. For instance, exogenously added cAMP restored the budding phenotype in a filamentous Ustilago maydis mutant with a defective adenylate cyclase that abolishes cAMP synthesis (Gold et al. 1994). In M. grisea, appressorium formation is induced by hydrophobicity and by exogenously added cAMP (Lee and Dean 1993). We found that appressorium formation can be induced by cAMP in the con 2 and con 4 mutants but not in the con 1 and con 7 mutants (Fig. 3). The con $2 /$ con 4 double mutant no longer responded to cAMP induction even though individ-

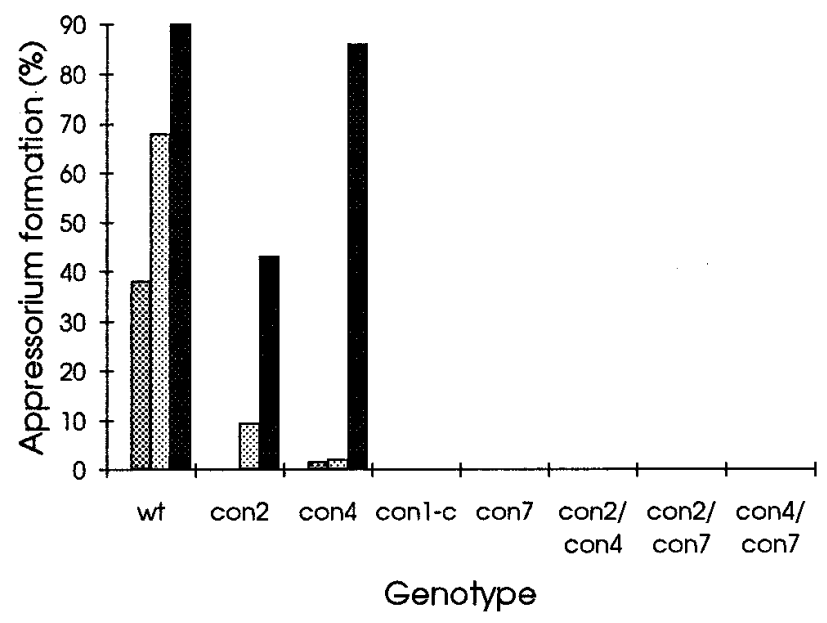

Fig. 3. Effect of exogenously added cyclic AMP on appressorium formation of single and double mutants of Magnaporthe grisea. Wild-type Guy11 shows increasing response throughout the range of concentrations tested (heavily dotted bar, $0.5 \mathrm{mM}$; lightly dotted bar, $5 \mathrm{mM}$; solid bar, $50 \mathrm{mM}$ ). Single mutants con2 and con 4 show a slight response at concentrations of 5 and $0.5 \mathrm{mM}$, respectively. Single mutants of conl and $\operatorname{con} 7$ and their respective double mutants are nonresponsive.

Table 3. Growth and sporulation of representative double mutants and ectopic transformants of Magnaporthe grisea ${ }^{\mathrm{a}}$

\begin{tabular}{|c|c|c|c|c|c|c|}
\hline \multirow{2}{*}{$\begin{array}{l}\text { Recipient strain } \\
\text { + plasmid }\end{array}$} & \multirow{2}{*}{$\begin{array}{c}\text { Plasmid } \\
\text { integration }\end{array}$} & \multirow[b]{2}{*}{ Strain } & \multirow[b]{2}{*}{ Mutation } & \multirow{2}{*}{$\begin{array}{l}\text { Radial growth } \\
\quad(\mathbf{m m})\end{array}$} & \multicolumn{2}{|c|}{ Sporulation $\left(\times 10^{4} / \mathrm{ml}\right)$} \\
\hline & & & & & Light & Dark \\
\hline \multirow{2}{*}{ GR43-3+pGT20A } & Replacement & GR44-1 & con $4 / \operatorname{con} 7$ & $37 \pm 1.4^{\mathrm{b}}$ & $43.9 \pm 8.0$ & $46.8 \pm 8.0$ \\
\hline & Ectopic & GR44-10 & $\operatorname{con} 7$ & $36.5 \pm 1.3$ & $278.1 \pm 14.9$ & $273.8 \pm 39.1$ \\
\hline \multirow[t]{3}{*}{ Guy11-con2 + pGT20A } & None & Guy11-con2 & con 2 & $42.8 \pm 0.6$ & $5.5 \pm 1.3$ & 0 \\
\hline & Replacement & GR14-3 & $\operatorname{con} 2 / \operatorname{con} 4$ & $41.9 \pm 0.5$ & $12.6 \pm 5.4$ & $0.3 \pm 0.5$ \\
\hline & Ectopic & GR14-1 & con 2 & $36 \pm 0$ & $14 \pm 3.1$ & $0.3 \pm 0.5$ \\
\hline Guy11 + pAN7-2 & Insertion & GT20 & con4 & $37.1 \pm 0.4$ & $158.2 \pm 13.0$ & $1.1 \pm 0.4$ \\
\hline \multirow[t]{2}{*}{ Guy $11-\operatorname{con} 2+$ pGT562A } & Replacement & GR19-8 & $\operatorname{con} 2 / \operatorname{con} 7$ & $38.3 \pm 0.5$ & $1.6 \pm 0.7$ & $0.13 \pm 0.2$ \\
\hline & Ectopic & GR19-7 & con 2 & $41.6 \pm 0.7$ & $21.3 \pm 5.5$ & $0.3 \pm 0.6$ \\
\hline \multirow[t]{3}{*}{ 89-8-1+ pGT20A } & None & $89-8-1$ & conl-C & $41.2 \pm 0.5$ & $1.6 \pm 0.4$ & $0.8 \pm 0.2$ \\
\hline & Replacement & GR28-4 & con1-C/con4 & $24.2 \pm 0.5$ & 0 & 0 \\
\hline & Ectopic & GR28-9 & con $1-C$ & $42.0 \pm 0.0$ & $1.8 \pm 0.5$ & $0.4 \pm 0.1$ \\
\hline \multirow{2}{*}{ 89-8-1 + pGT562A } & Replacement & GR29-1 & con $1-C / \operatorname{con} 7$ & $41.5 \pm 1.0$ & 0 & 0 \\
\hline & Ectopic & GR29-9 & con $1-C$ & $41.1 \pm 1.0$ & $2.5 \pm 0.7$ & $0.3 \pm 0.1$ \\
\hline
\end{tabular}

\footnotetext{
${ }^{a}$ Vegetative growth was measured at ninth day before sporulation was assayed by washing cultures in a 6-cm petri plate with $1 \mathrm{ml}$ of sterile water.
}

b \pm standard deviation. 
ual parental mutants responded at a reduced level. These data suggest that con 2 and con 4 mutations may be influenced directly or indirectly by the cAMP signaling pathway, whereas con 7 and conl are not.

Pathogenicity tests of double mutants show additive as well as epistatic interactions between the mutations. The original con 2 and con 4 mutants are weakly pathogenic to rice (Shi and Leung 1995). The con $2 /$ con4 mutants, however, produced only a few lesions with spray and wound inoculation even when a high inoculum intensity $\left(5 \times 10^{6}\right.$ spores $)$ was used, suggesting that the accumulated developmental defects have led to a further reduction in pathogenicity in the con $2 /$ con 4 mutant. In contrast, the con 7 mutation is pathogenically epistatic to con 2 and con4 (Fig. 4B). There was no sign of infection with the con $2 / \operatorname{con} 7$ and con $4 / \operatorname{con} 7$ mutants when plants were spray or wound inoculated with a high level of inoculum $\left(10^{5}\right.$ to $10^{6}$ spores). That epistatic relationship is dependent on the phenotypes assayed has been demonstrated in A. nidulans (Martinelli 1979). For instance, the bristle mutation is epistatic to the medusa mutation in conidiophore structure, yet the medusa mutations are epistatic to a leaky bristle mutation in pigmentation. The different phenotypic expressions of gene interactions observed in M. grisea suggest that each gene has multiple functions in different developmental processes and, consequently, their interactions lead to diverse phenotypic expression.

The epistatic relationships described in this paper are based on the assumption that the parental mutants (Guy11-con2, 898-1, and GR43-1) used as transformation recipients and the two retrieved plasmids used for gene disruption each contain a single mutated gene. Our genetic and molecular analyses conducted on these mutants, so far, support this assumption (Shi and Leung 1995; Z. Shi and H. Leung, unpublished data). The gene disruption experiments conducted with the retrieved con4 and con 7 genes (pGT20A and pGT562A) further support this conclusion. It is possible, however, that one mutation effects two or more genes that are genetically inseparable. Fur- thermore, the epistatic relationships described in this paper are based on the phenotypic characterization of double mutants, which reflects our current knowledge of the developmental processes of sporulation, appressorium formation, and pathogenesis. A molecular understanding of the interactions among these genes will require a full characterization of the genes and their gene products.

In conclusion, we have demonstrated that morphogenetic genes have multiple functions, and hence their interactions result in epistatic relationships unique to a specific develop-
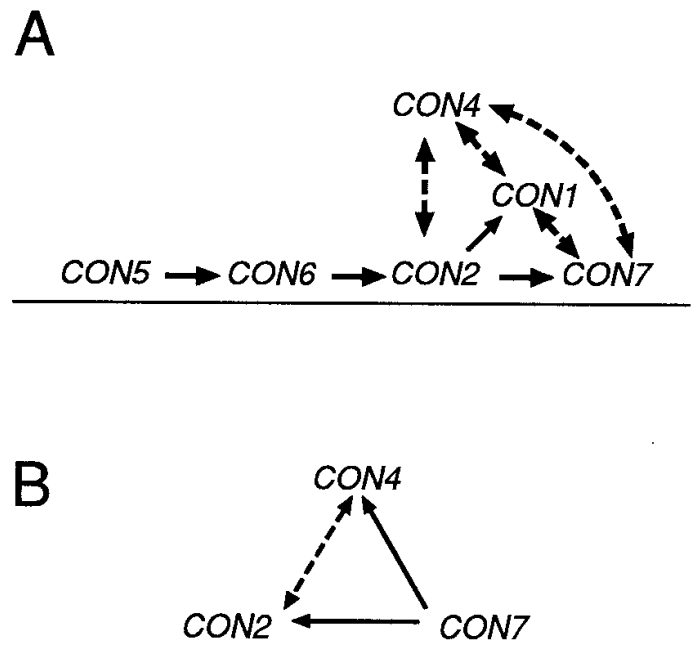

Fig. 4. A, Model depicting epistatic and interactive relationships among morphogenetic loci in conidiogenesis in Magnaporthe grisea. Solid arrows indicate direction of epistasis; broken two-way arrows indicate interactions producing hybrid phenotypes. con 2 acts at a branch point before con1 and con7. con4 acts independently of the core pathway, but strongly influences spore morphogenesis. Disruption of conl, along with either $\operatorname{con} 4$ or $\operatorname{con} 7$, suppresses the formation of conidia. B, The epistatic relationships change; however, when pathogenicity is considered. con 7 becomes epistatic to both con 2 and con4.

Table 4. Pathogenicity of representative double mutants, ectopic transformants, and recipient strains of Magnaporthe grisea

\begin{tabular}{|c|c|c|c|c|c|}
\hline \multirow[b]{2}{*}{ Strain } & \multirow[b]{2}{*}{ Integrated plasmid } & \multirow[b]{2}{*}{ Genotype } & \multicolumn{2}{|c|}{ Lesions per leaf of M103 (no.) at two inoculum levels ${ }^{\mathrm{a}}$} & \multirow[b]{2}{*}{ Wound inoculation $^{\mathrm{h}}$} \\
\hline & & & $5 \times 10^{5}$ & $5 \times 10^{6}$ & \\
\hline GR17-1 & pGT20A & con4 & 0 & $0.8 \pm 1.3$ & + \\
\hline GR17-3 & pGT20A & wild type & $6.4 \pm 1.7$ & $19.2 \pm 2.5$ & + \\
\hline GR17-4 & pGT20A & wild type & $3.3 \pm 1.9$ & $11.5 \pm 0.9$ & + \\
\hline GR14-3 & pGT20A & $\operatorname{con} 2 / \operatorname{con} 4$ & 0 & $0.4 \pm 0.6$ & + \\
\hline GR14-7 & pGT20A & $\operatorname{con} 2 / \operatorname{con} 4$ & 0 & $0.3 \pm 0.7$ & + \\
\hline GR16-17 & pGT562A & $\operatorname{con} 7$ & 0 & 0 & - \\
\hline GR16-35 & pGT562A & $\operatorname{con} 7$ & 0 & 0 & - \\
\hline GR16-3 & pGT562A & Wild type & $3.4 \pm 0.5$ & $15.0 \pm 5.2$ & + \\
\hline GR16-4 & pGT562A & Wild type & $9.2 \pm 2.2$ & $11.0 \pm 1.7$ & + \\
\hline GR19-7 & pGT562A & $\operatorname{con} 2$ & $0.1 \pm 0.2$ & $4 \pm 3.4$ & + \\
\hline GR19-8 & pGT562A & $\operatorname{con} 2 / \operatorname{con} 7$ & 0 & 0 & - \\
\hline GR44-10 & pGT20A & $\operatorname{con} 7$ & 0 & 0 & - \\
\hline GR44-1 & pGT20A & $\operatorname{con} 4 / \operatorname{con} 7$ & 0 & 0 & - \\
\hline GR44-3 & pGT20A & $\operatorname{con} 4 / \operatorname{con} 7$ & 0 & 0 & - \\
\hline Guy11-con2 & None & $\operatorname{con} 2$ & $0.2 \pm 0.4$ & $1.6 \pm 1.9$ & + \\
\hline GT20 & pAN7-2 & $\operatorname{con} 4$ & $0.2 \pm 0.4$ & $0.3 \pm 0.6$ & + \\
\hline GT562 & pAN7-2 & $\operatorname{con} 7$ & 0 & 0 & - \\
\hline GR43-3 & pCON7::bar & $\operatorname{con} 7$ & 0 & 0 & - \\
\hline Guy11 & None & Wild type & $21.5 \pm 9.3$ & $>30$ & + \\
\hline
\end{tabular}

a Total number of spores delivered in $8 \mathrm{ml}$ of spore suspension. Approximately 10 to 15 plants were inoculated with each strain. Inoculation was conducted at least twice. \pm standard deviation.

$\mathrm{b}+=$ lesions observed, $-=$ no lesion. 
mental process. There exists a core pathway determining the progression of conidial development yet other genes can exert strong effects on the morphogenetic process. Double mutations in different pathways can lead to changes in spore morphology, suppression of sporulation, and reduced pathogenicity. A practical objective in our investigation of sporulation in M. grisea is to identify genes or developmental processes as targets for intervention. This strategy requires a clear understanding of the degree of autonomy of a developmental pathway. The suppression of spore formation and pathogenesis shown in double mutants suggests that it may be possible to identify multiple sites for intervention.

\section{MATERIALS AND METHODS}

\section{Strains and media.}

Characteristics of the $M$. grisea strains and plasmids used and generated in this study are listed in Table 1 . Transformants generated from gene disruption experiments were designated as the GR series. Cultures were maintained on oatmeal agar and colonized paper disks as described (Leung et al. 1988). E. coli strains DH5 $\alpha$ and DH10B were used for propagation of plasmid DNA. E. coli harboring plasmids were grown in Luria-Bertani (LB) medium supplemented with the appropriate antibiotics (Sambrook et al. 1989).

\section{Retrieval of the mutated $\mathrm{CON} 4$ and $\mathrm{CON7}$ loci.}

Genomic DNA of the GT20 (con4) and GT562 (con7) mutants was isolated by the CTAB method as described (Borromeo et al. 1993). Fungal genomic sequences flanking the inserted plasmid in GT20 and GT562 were obtained by plasmid rescue as described (Kuspa and Loomis 1992). Briefly, approximately $0.5 \mu \mathrm{g}$ of DNA was digested with ApaI, which does not cut within pAN7-2. The digested DNA was ethanol precipitated, dried, and redissolved in Tris-EDTA. The DNA was circularized in a 500- $\mu$ l ligation reaction containing 5 units of T4 DNA ligase, and incubated at $14^{\circ} \mathrm{C}$ overnight. Ligated DNA was precipitated and redissolved in sterile water to a final concentration of $0.1 \mu \mathrm{g} / \mu \mathrm{l}$. One microliter of the ligated DNA was used to transform $E$. coli DH10B cells by electroporation with a cell porater (Cell-Porator E. coli Electroporation System, Gibco BRL, Gaithersburg, MD). E. coli transformants were selected on LB agar plates containing ampicillin $(50 \mu \mathrm{g} / \mathrm{ml})$ and the plasmids were recovered by the alkaline lysis method as described (Sambrook et al. 1989). The retrieved plasmids were analyzed by restriction mapping to confirm that they contained the original pAN7-2 plus genomic sequences presumably representing the mutated genes in the mutants. The retrieved plasmids were used for gene disruption.

\section{Transformation.}

The plasmids containing the inactivated genes (con4 or con7) were linearized with $A p a I$ and introduced into Guy11 (wild type), Guy11-con2 (con2), 89-8-1 (con1-C), and GR433 (con7) by the transformation procedure described by Leung et al. (1990) and Shi et al. (1995). Hygromycin B resistant transformants were transferred to oatmeal agar and incubated under direct illumination $\left(140 \mu \mathrm{E} \cdot \mathrm{s}^{-1} \cdot \mathrm{m}^{-2}\right)$ for 5 days to induce sporulation. Conidial morphology and colony morphology were examined by light microscopy to identify genedisrupted mutants. Putative gene-disruption mutants and ectopic transformants with recipient strain phenotypes were subjected to phenotypic, genetic, and molecular analyses.

\section{Analysis of transformants.}

Transformants with novel conidial and colony morphology were purified by single sporing to confirm the mutant phenotypes and then crossed to 2539 to determine their inheritance by standard crossing methods (Leung and Williams 1985). Segregation patterns and linkage relationship between two genes were tested by Chi-square goodness-of-fit test. Genedisrupted mutants and representative recipient type transformants were analyzed by Southern hybridization to determine whether the mutant phenotypes were the result of gene replacement at the corresponding loci. DNA was digested with EcoRI or BamHI, electrophoresed, and blotted onto nylon membrane (Hybond N-plus, Amersham, Chicago) according to manufacturer's instructions. pAN7-2 was labeled with ${ }^{32} \mathrm{P}$ dATP (DECAprime DNA Labeling Kit, Ambion, Austin, TX) and used to probe the genomic blots. Hybridization signals were detected by standard autoradiography (Sambrook et al. 1989).

Confirmed gene-replaced mutants and recipient type transformants (ectopic integrants) were examined for conidial morphology by differential interference contrast microscopy. Appressorium formation, growth, and sporulation were assayed as described by Shi and Leung (1995). The response of the mutants to cAMP (Sigma Chemical, St. Louis, MO) was tested by a modified procedure described by Lee and Dean (1993). Microscope coverslips were soaked in $95 \%$ ethanol, then flamed, to make them hydro-

Table 5. Summary of epistatic relationships between gene pairs in Magnaporthe grisea

\begin{tabular}{|c|c|c|c|c|c|c|}
\hline \multirow[b]{2}{*}{ Mutant genotype } & \multicolumn{2}{|c|}{ Morphology } & \multirow{2}{*}{$\begin{array}{l}\text { Light response } \\
\text { of sporulation }\end{array}$} & \multirow{2}{*}{$\begin{array}{c}\text { Appressorium } \\
\text { formation }\end{array}$} & \multirow[b]{2}{*}{ Cyclic AMP response } & \multirow[b]{2}{*}{ Pathogenicity } \\
\hline & spore & colony $^{b}$ & & & & \\
\hline $\operatorname{con} 1 / \operatorname{con} 4$ & $\mathrm{NA}^{\mathrm{c}}$ & Additive $^{\mathrm{d}}$ & NA & NA & NA & NA \\
\hline $\operatorname{con} 1 / \operatorname{con} 7$ & NA & Hybrid & NA & NA & NA & NA \\
\hline $\operatorname{con} 2 / \operatorname{con} 4$ & Hybrid $^{\mathrm{e}}$ & Hybrid & Hybrid & Additive & Additive & Additive \\
\hline $\operatorname{con} 2 / \operatorname{con} 7$ & $\operatorname{con} 2>\operatorname{con} 7^{\mathrm{f}}$ & $\operatorname{con} 7>\operatorname{con} 2$ & $\operatorname{con} 2>\operatorname{con} 7$ & $\operatorname{con} 7>\operatorname{con} 2$ & $\operatorname{con} 7>\operatorname{con} 2$ & $\operatorname{con} 7>\operatorname{con} 2$ \\
\hline $\operatorname{con} 4 / \operatorname{con} 7$ & Hybrid & $\operatorname{con} 7>\operatorname{con} 4$ & $\operatorname{con} 7>\operatorname{con} 4$ & $\operatorname{con} 7>\operatorname{con} 4$ & $\operatorname{con} 7>\operatorname{con} 4$ & $\operatorname{con} 7>\operatorname{con} 4$ \\
\hline
\end{tabular}

\footnotetext{
${ }^{a}$ Epistatic relationships are determined based on the phenotypes of double mutants in respective developmental processes.

${ }^{\mathrm{b}}$ Features that determine an overall colony appearance include vegetative growth, pigmentation, abundance of mycelia, and conidiophores.

c Not applicable.

${ }^{\mathrm{d}}$ Additive effect of two mutated genes was observed in the double mutant. In all cases, the defect was more pronounced than in the single-gene mutants.

e Hybrid phenotype.

$\mathrm{f}>$ indicates direction of epistasis.
} 
philic. A $20-\mu \mathrm{l}$ spore suspension (approximately $10^{4}$ spores/ $\mathrm{ml}$ ) in different concentrations of cAMP (water control, 0.5 $\mathrm{mM}, 5 \mathrm{mM}$, and $50 \mathrm{mM}$ ) was placed on a sterile coverslip. The samples were incubated in a moist chamber for $24 \mathrm{~h}$ and then inverted (with the drop of spore suspension facing down) and placed on a microscope slide. The proportion of germ tubes forming appressoria was determined by counting approximately 100 germlings per slide. Appressorium formation was also observed on excised leaf sheaths, affixed to microscope slides with "double coated" tape, by spotting spore suspension (approximately $10^{5}$ spores per ml) onto the tissue and incubating in a moist chamber for $24 \mathrm{~h}$. Appressorium formation was observed microscopically. Experiments were conducted at least three times. Pathogenicity of transformants and the original mutants was tested on rice variety M103 (provided by David MacKill, University of California-Davis). Plants were inoculated by spraying and leaf-sheath injection and the number of lesions recorded as described (Shi and Leung 1994). Approximately 10 to 15 plants were inoculated with each strain per experiment and at least two independent inoculation tests were conducted.

\section{ACKNOWLEDGMENTS}

We thank Peter Punt for providing pAN7-2, Martin Pall for providing pBARGEM7-2, Albert Ellingboe for providing strain 89-8-1, and Dave MacKill for providing rice seed. Comments on the manuscript by several reviewers are greatly appreciated. This work was supported in part by a grant from the Rockefeller Foundation Rice Biotechnology Program and U.S. Department of Agriculture NRI grant. Contribution PPNS 0261 from Washington State Agricultural Research Center.

\section{LITERATURE CITED}

Borromeo, E. S., Nelson, R. J., Bonman, J. M., and Leung, H. 1993. Genetic differentiation among isolates of Pyricularia grisea infecting rice and weed hosts. Phytopathology 83:393-399.

Busby, T. M., Miller, K. Y., and Miller, B. L. 1996. Suppression and enhancement of the Aspergillus nidulans medusa mutation by altered dosage of the bristle and stunted genes. Genetics 143:155-163.

Gold, S. E., Duncan, G. A., Barrett, K. J., and Kronstad, J. W. 1994. cAMP regulates morphogenesis in the pathogenic fungus Ustilago maydis. Genes Dev. 8:2805-2816.

Kuspa, A., and Loomis, W. F. 1992. Tagging developmental genes in
Dictyostelium by restriction enzyme-mediated integration of plasmid DNA. Proc. Natl. Acad. Sci. USA 89:8803-8807.

Lee, Y. H., and Dean, R. A. 1993. Cyclic AMP regulates infection structure formation by the plant pathogenic fungus Magnaporthe grisea. Plant Cell 5:693-700.

Leung, H., Borromeo, E. S., Bernardo, M. A., and Notteghem, J. L. 1988. Genetic analysis of virulence in the rice blast fungus Magnaporthe grisea. Phytopathology 78:1227-1233.

Leung, H., Lehtinen, U., Karjarlainen, R., Skinner, D. Z., Tooley, P. W., Leong, S. A., and Ellingboe, A. H. 1990. Transformation of the rice blast fungus Magnaporthe grisea to hygromycin B resistance. Curr. Genet. 17:409-411.

Leung, H., and Shi, Z. 1994. Genetic regulation of sporulation in the rice blast fungus. Pages 35-50 in: Rice Blast Disease. R. S. Zeigler, S. A. Leong, and P. S. Teng, eds. CAB International, Wallingford, Oxon, UK.

Leung, H., and Williams, P. H. 1985. Genetic analyses of electrophoretic enzyme variants, mating type, and hermaphroditism in Pyricularia oryzae Cavara. Can. J. Genet. Cytol. 27:697-704.

Martinelli, S. D. 1979. Phenotypes of double conidiation mutants of Aspergillus nidulans. J. Gen. Microbiol. 114:277-287.

Miller, B. L. 1990. The developmental genetics of asexual reproduction in Aspergillus nidulans. Semin. Dev. Biol. 1:207-219.

Pall, M. L., and Brunelli, J. P. 1993. A series of six compact fungal transformation vectors containing polylinkers with multiple unique restriction sites. Fungal Genet. Newsl. 40:59-62.

Parsons, K. A., Chumley, F. G., and Valent, B. 1987. Genetic transformation of the fungal pathogen responsible for rice blast disease. Proc. Natl. Acad. Sci. USA 84:4161-4165.

Punt, P. J., Oliver, R. P., Dingemanse, M. A., Pouwels, P. H., and Van den Hondel, C. A. M. J. J. 1987. Transformation of Aspergillus based on the hygromycin B resistance marker from Escherichia coli. Gene 56:117-124.

Sambrook, J., Fritsch, E. F., and Maniatis, T. A. 1989. Molecular Cloning: A Laboratory Manual. 2nd ed. Cold Spring Harbor Laboratory, Cold Spring Harbor, NY.

Shi, Z., Christian, D., and Leung, H. 1995. Enhanced transformation in Magnaporthe grisea by restriction enzyme mediated integration of plasmid DNA. Phytopathology 85:329-333.

Shi, Z., and Leung, H. 1994. Genetic analysis and rapid mapping of a sporulation mutation in Magnaporthe grisea. Mol. Plant-Microbe Interact. 7:113-120.

Shi, Z., and Leung, H. 1995. Genetic analysis of sporulation in Magnaporthe grisea by chemical and insertional mutagenesis. Mol. PlantMicrobe Interact. 8: 949-959.

Timberlake, W. E. 1993. Translational triggering and feedback fixation in the control of fungal development. Plant Cell 5:1453-1460.

Valent, B., and Chumley, F. G. 1991. Molecular genetic analysis of the rice blast fungus, Magnaporthe grisea. Annu. Rev. Phytopathol. 29: 443-467. 